\title{
NCACO-score: An effective main-chain dependent scoring function for structure modeling
}

Liqing Tian ${ }^{1,2+}$, Aiping $\mathrm{Wu}^{1 \dagger}$, Yang Cao ${ }^{1,2}$, Xiaoxi Dong ${ }^{1,2}$, Yun $\mathrm{Hu}^{1,2}$ and Taijiao Jiang ${ }^{1 *}$

\begin{abstract}
Background: Development of effective scoring functions is a critical component to the success of protein structure modeling. Previously, many efforts have been dedicated to the development of scoring functions. Despite these efforts, development of an effective scoring function that can achieve both good accuracy and fast speed still presents a grand challenge.

Results: Based on a coarse-grained representation of a protein structure by using only four main-chain atoms: N, $\mathrm{C} \alpha, \mathrm{C}$ and $\mathrm{O}$, we develop a knowledge-based scoring function, called NCACO-score, that integrates different structural information to rapidly model protein structure from sequence. In testing on the Decoys'R'Us sets, we found that NCACO-score can effectively recognize native conformers from their decoys. Furthermore, we demonstrate that NCACO-score can effectively guide fragment assembly for protein structure prediction, which has achieved a good performance in building the structure models for hard targets from CASP8 in terms of both accuracy and speed.

Conclusions: Although NCACO-score is developed based on a coarse-grained model, it is able to discriminate native conformers from decoy conformers with high accuracy. NCACO is a very effective scoring function for structure modeling.
\end{abstract}

\section{Background}

A central stage at the protein structure modeling is to develop an effective energy function, also called potential or scoring function, which generally fall into two categories: physical-based and knowledge-based energy functions. Physical-based energy functions are derived from the laws of physics, which often use molecular mechanics method [1-3]. Whereas, knowledge-based energy functions are based on statistical analysis of experimentally determined protein structures, which provide an excellent shortcut towards a powerful energy function [4]. Compared to physical-based energy functions, knowledge-based energy functions have become more and more popular in protein structure prediction due to the relatively easy generation and manipulation of model structures and the lower computational cost. This can be seen from recent CASPs (Critical

\footnotetext{
* Correspondence: taijiao@moon.ibp.ac.cn

† Contributed equally

${ }^{1}$ National Laboratory of Biomacromolecules, Institute of Biophysics, Chinese Academy of Sciences, Beijing 100101, China

Full list of author information is available at the end of the article
}

Assessment of Techniques for Protein Structure Prediction), in which the most successful prediction methods use knowledge-based energy functions [5,6]. Moreover, knowledge-based approaches have also been widely used in protein design [7], validation of experimentally determined protein structures $[8,9]$ and protein-protein and protein-ligand interactions [10].

Knowledge-based energy functions can also be developed at different level of complexity depending on the level of structural details considered in the structural models. At the most detailed structural level, the knowledge-based energy functions require the structural information of all heavy atoms [4,11-19]. The all-atom knowledge-based energy functions can achieve good accuracy in structural analysis, but they typically incur intensive computation [20]. To reduce running time, many efforts have attempted to develop coarse-grained models with reduced structural representation [21-32]. The simplest coarse-grained model is to represent a residue as a point and thus a protein structure can be delineated as a simple $\mathrm{C}_{\alpha}$-trace. The coarse-grained models at residue level indeed can significantly decrease 
the running time in structure modeling but they usually suffer unsatisfactory accuracy [21].

To balance the accuracy and computational time, the intermediate models between the atom-level and residue-level representations have also been developed $[15,21,22,24]$. In these models, the side chains are considered, which are usually simplified as $C_{\beta}$ atoms or the side chain center of mass. For example, in low resolution structure prediction, the Rosetta of Baker lab used a structural model represented by heavy main-chain atoms and pseudo side chain center of mass [33]. More recently, Makino and Itoh also developed a knowledgebased potential named DFMAC that requires the coordinates of main-chain atoms $\left(\mathrm{N}, \mathrm{C}_{\alpha}\right.$ and $\left.\mathrm{C}\right)$ and pseudo $\mathrm{C}_{\beta}$ atom [21]. Since the side chain conformations have been abstracted, these main-chain dependent models can achieve both relatively good accuracy and reasonable running time. Although these energy models have showed high ability of structure discrimination on known decoy data sets, few have demonstrated success in structure modeling, especially in de novo structure prediction. This is because de novo structure prediction requires high-performance scoring function not only with high accuracy of structure discrimination at low computational expense, but also with a smooth energy landscape which goes beyond the ability to distinguish the known decoy data sets. For example, DFMAC uses an orientation-dependent potential term between two $\mathrm{C}_{\alpha}$-pseudo- $\mathrm{C}_{\beta}$ vectors. Although the orientation-dependent potential showed a considerable improvement in distinguishing native structures and non-native (or decoy) structures, it relies on strict geometry features which are difficult to be satisfied due to the inaccuracy of pseudo atoms. This could significantly affect its use in structural modeling. Therefore, development of scoring functions for effective structure modeling still remains a challenging problem.

Here we also attempt to develop an effective knowledge-based scoring function based on main-chain dependent coarse-grained model that only requires the main-chain atoms $\left(\mathrm{N} \mathrm{C}_{\alpha} \mathrm{C}\right.$ and $\left.\mathrm{O}\right)$ coordinates per residue, which we called as NCACO-score. In developing $\mathrm{NCACO}$-score, we considered an integration of four potential terms: contact-based pairwise atom-atom interactions, sequence-dependent local conformational propensities, solvation effects, and geometry propensities of pairwise triplet fragments on beta sheet. Although the first three terms follow the same form of other knowledge-based scoring functions $[11,21]$, they are derived differently and carefully parameterized in the integration (see Methods). Moreover, we proposed a new potential term, the fourth term, to promote the formation of beta sheet during the structure modeling. The testing on 32 decoy sets from Decoys'R'Us database [34] shows that
NCACO-score outperforms other coarse-grained potentials in discriminating native structures from their decoy structures. Remarkably, its average Z-score is much lower than those other coarse-grained potentials, indicating that $\mathrm{NCACO}$-score has a larger energy gap between the native state and nonnative ones. This suggests its potential and effectiveness in structure prediction [13]. Furthermore, in an application of the scoring function to structure prediction using a simple fragment-based assembly procedure, we found that the structures modeled for the 14 hard targets of CASP8 achieved comparable accuracy with those predicted by Robetta [35]. The average running time of prediction for these targets was $\sim 64.5 \mathrm{~h}$ of CPU time. This demonstrates the effectiveness of the NCACO-score in structure modeling. We believe that the scoring function could be very useful for protein structure modeling.

\section{Results}

\section{Overview of NCACO-score}

In NCACO-score, we consider two levels of coarsegrained structure representations. One is a five-bead model, in which a residue is represented as four mainchain atoms ( $\mathrm{N} \mathrm{CaC}$ and $\mathrm{O}$ ) and a pseudo side chain center of mass (except GLY). The pseudo Side chain Center of Mass (SCM) was determined according to the $\phi / \psi$ backbone torsion angles of the residue. We use the five-bead model to derive pairwise atom-atom contact potential $\left(E_{c o n}\right)$ and sequence-dependent local conformational potential $\left(E_{t r p}\right)$. The other is a residue-level structure representation requiring $\mathrm{C} \alpha$ coordinates only. We use the residue-level model to derive solvation potential $\left(E_{s o l}\right)$ and beta sheet geometry propensity potential $\left(E_{\text {beta }}\right)$. Therefore, our NCACO-score integrates the above four terms:

$$
E_{\text {tot }}=E_{\text {con }}+5.4 E_{\text {trp }}+12.3 E_{\text {sol }}+3.1 E_{\text {beta }}
$$

The potential components considered in NCACOscore could reflect different aspects of protein folding principle. $E_{c o n}$ stands for the mean interaction potential between two atoms, which reflects the compatibility of the whole protein structure. This potential is the most widely used term in knowledge-based scoring function, and is even used alone in some pioneers' work $[13,15,28,32] . E_{t r p}$ reflects the local conformational biases of secondary structure, which is local-sequence dependent. $E_{\text {sol }}$ stands for the solvation energy of protein, which reflects the hydrophobic effect of protein folding. We used a simple model based on $\mathrm{C} \alpha$ atom to derive the solvation energy by following Makino and Itoh's method [21]. To promote the formation of beta sheet, we introduced $E_{\text {beta }}$ to capture the geometrical features of beta sheet at residue level. The details of these four terms and their integration are described in 
Methods. Although it's difficult to construct orthogonal potential components for knowledge-based scoring function, we will show below that the integration of these four terms indeed improves structure discrimination ability, enabling us to develop an effective knowledgebased scoring function.

\section{Assessment of structure discrimination ability of NCACO- score and its individual terms}

To gain insights into how well NCACO-score and its individual components discriminate native structures from non-native structures (also called decoys), we looked into their performance of structure discrimination on the Decoys'R'Us sets. 32 proteins from five decoy sets were selected: 1 ) 4state_reduced (seven proteins), 2) fisa (four proteins), 3) fisa_casp3 (three proteins), 4) lmds (ten proteins), and 5) lattice_ssfit (eight proteins).

Table 1 summarizes the discrimination power of NCACO-score and its four individual potential terms on the five decoy sets in terms of the ranks and Z-scores of native structures. As shown in the table, NCACO-score ranked the first the native structures of 23 proteins out of 32 proteins. Remarkably, the Z-scores of native structures were very low in most of the 32 proteins with average Z-score of -5.06 , demonstrating the great structure discrimination ability of NCACO-score. Despite its overall good performance, NCACO-score could not effectively discriminate between the native and decoy structures for the two proteins ( $1 \mathrm{fc} 2$ in fisa set, and 1bba in lmds set) (z-score $>0$ ), which we will explain below.

For individual terms, $E_{t r p}$ had the best performance, which ranked the first 21 of 32 native structures with average Z-score of -5.34, indicating that it contributed the most to NCACO-score. Although $E_{c o n}$ and $E_{s o l}$ overall had lower ability of structure discrimination compared to $E_{t r p}$, they outperformed $E_{t r p}$ in the fisa set. $E_{\text {beta }}$, which is designed to capture the structural feature of beta sheet, indeed showed a good performance for most proteins containing beta sheet (average Z-score = -6.92).

From above, we can see that the performance of NCACO-score largely relies on the individual term $E_{t r p}$. Therefore, the extremely poor performance of $E_{t r p}$ in discriminating the native and decoy structures of four proteins ( $1 \mathrm{fc} 2$ and 1 hdd-C in fisa set, $1 \mathrm{bba}$ and $1 \mathrm{fc} 2$ in lmds set) could significantly affect the performance of NCACO-score in these four proteins. However, $E_{c o n}$ and $E_{\text {sol }}$ have better structure discrimination than $E_{t r p}$ in these four proteins. As shown in Table 1, the integration of these two terms improves the performance NCACOscore, particularly for 1 hdd-C in fisa set and $1 \mathrm{fc} 2$ in lmds set, in which integrated NCACO-score significantly outperforms $E_{t r p}$. This suggests the necessity of the integration of these different terms in developing an effective scoring function.

The decoy discrimination ability of NCACO-score was also rigorously evaluated using 4-fold cross-validations on the Decoys'R'Us sets (see additional file 1). As seen from the results of cross-validations, NCACO-score performed nearly equally well in both training sets and testing sets not only for the average rank of native structures (38.44 in training sets vs 36.96 in testing sets) but also for the Z-score of native structures $(-5.10$ in training sets vs -4.93 in testing sets). The close performance of our model in training sets and testing sets suggests the robustness and reliability of our model.

A good energy function should be able to discriminate native structure from near-native decoys [36]. However, Decoys'R'Us sets are not suitable for this test, because most of the decoys are far from native structures. Therefore, we further generated a new near-native decoy set by fragment replacement method (see additional file 2). This data set consists of 87 proteins with decoys of TM-scores between 0.6 and 0.9 compared to the native structure (see additional file 2). When NCACO-score was tested on this data set, the average Pearson correlation coefficient between energy and cRMSD for the 87 proteins was 0.64 (see additional file 2), demonstrating the reliability of NCACO-score in discrimination of near native structures.

\section{Comparison with other knowledge-based scoring functions}

We compared the performance of NCACO-score on the 32 Decoys'R'Us sets with 6 state-of-the-art knowledgebased scoring functions: RAPDF [19], Atomic KBP [13], DFIRE-A [15], DFIRE-B [15], PC2CA [22], and DFMAC [21]. Table 2 shows comparison of the ranks and Zscores of the native structures. RAPDF, Atomic KBP, and DFIRE-A need coordinates of all heavy atoms of main chain and side chain. DFIRE-B needs information of main chain and $C_{\beta}$. PC2CA needs information of $C_{\alpha}$ and $C_{\beta}$. DFMAC needs information of the main-chain atoms $\left(\mathrm{N}, \mathrm{C}_{\alpha}\right.$, and $\left.\mathrm{C}\right)$. Like DFMAC, our scoring function NCACO-score needs coordinates of the main-chain atoms $\left(\mathrm{N}, \mathrm{C}_{\alpha}, \mathrm{C}\right.$, and $\left.\mathrm{O}\right)$. The comparison results showed NCACO-score had the best discrimination performance on the 32 proteins in terms of both the average rank and the average $\mathrm{Z}$-score. Among the four functions which used coarse-grained model (DFIRE-B, PC2CA, DFMAC, and NCACO-score), NCACO-score had the lowest average Z-score (-5.06), followed by DFMAC (-4.05), PC2CA (-3.48) and DFIRE-B (-3.32). For the average rank of native structure, NCACO-score also gave the best rank (36.84), followed by PC2CA (39.09) DFIRE-B (40.81) and DFMAC (47.53). 
Table 1 Performance of single terms and the total scoring function of NCACO-score on Decoys'R'Us sets.

\begin{tabular}{|c|c|c|c|c|c|c|c|c|c|c|c|}
\hline \multirow{2}{*}{ 4state_reduced } & \multirow[t]{2}{*}{ Size $^{a}$} & \multicolumn{2}{|c|}{$E_{\text {con }}$} & \multicolumn{2}{|c|}{$E_{t r p}$} & \multicolumn{2}{|c|}{$E_{\text {sol }}$} & \multicolumn{2}{|c|}{$E_{\text {beta }}$} & \multicolumn{2}{|c|}{$E_{\text {tot }}$} \\
\hline & & & & & & & & & & & \\
\hline $1 \mathrm{ctf}$ & 630 & $1^{b}$ & $-3.03^{c}$ & 1 & -5.62 & 3 & -1.92 & 3 & -4.46 & 1 & -5.48 \\
\hline 1 r69 & 676 & 2 & -2.72 & 1 & -4.94 & 29 & -1.64 & $-{ }^{d}$ & - & 1 & -4.47 \\
\hline $1 \mathrm{sn} 3$ & 660 & 1 & -2.52 & 1 & -9.58 & 175 & -0.68 & 46 & -1.51 & 1 & -4.73 \\
\hline $2 \mathrm{cro}$ & 673 & 17 & -1.99 & 1 & -4.74 & 46 & -1.42 & - & - & 1 & -4.36 \\
\hline $3 i c b$ & 654 & 47 & -1.42 & 1 & -3.89 & 21 & -1.48 & 59 & -0.97 & 1 & -3.33 \\
\hline 4pti & 686 & 9 & -2.34 & 1 & -9.51 & 55 & -1.30 & 147 & -0.62 & 1 & -6.10 \\
\hline $4 r \times n$ & 677 & 27 & -1.65 & 1 & -5.17 & 74 & -1.17 & 3 & -3.74 & 1 & -4.22 \\
\hline Average & & 14.86 & -2.24 & 1.00 & -6.21 & 57.57 & -1.37 & 51.60 & -2.26 & 1.00 & -4.67 \\
\hline \multicolumn{12}{|l|}{ Fisa } \\
\hline $1 \mathrm{fc} 2$ & 501 & 9 & -1.99 & 499 & 3.32 & 6 & -1.92 & - & - & 461 & 1.68 \\
\hline 1hdd-C & 501 & 3 & -3.08 & 375 & 0.61 & 1 & -4.27 & - & - & 21 & -1.55 \\
\hline $2 \mathrm{cro}$ & 501 & 13 & -2.10 & 16 & -1.74 & 56 & -1.21 & - & - & 3 & -2.43 \\
\hline $4 i c b$ & 500 & 1 & -3.30 & 4 & -2.28 & 2 & -3.14 & 1 & -42.13 & 1 & -4.43 \\
\hline Average & & 6.50 & -2.62 & 223.50 & -0.02 & 16.25 & -2.64 & 1.00 & -42.13 & 121.50 & -1.68 \\
\hline \multicolumn{12}{|c|}{ fisa_casp3 } \\
\hline 1bg8-A & 1200 & 377 & -0.47 & 8 & -2.23 & 850 & 0.55 & - & - & 44 & -1.69 \\
\hline $1 \mathrm{bl0}$ & 972 & 818 & 1.02 & 1 & -4.30 & 730 & 0.60 & - & - & 3 & -2.46 \\
\hline 1jwe & 1407 & 387 & -0.64 & 6 & -2.66 & 343 & -0.80 & - & - & 6 & -2.33 \\
\hline Average & & 527.33 & -0.03 & 5.00 & -3.06 & 641.00 & 0.12 & - & - & 17.67 & -2.16 \\
\hline \multicolumn{12}{|l|}{ Imds } \\
\hline $1 b 0 n-B$ & 498 & 4 & -2.54 & 17 & -1.99 & 136 & -0.56 & - & - & 1 & -2.56 \\
\hline $1 \mathrm{bba}$ & 501 & 294 & 0.26 & 498 & 1.69 & 348 & 0.43 & - & - & 497 & 2.02 \\
\hline $1 \mathrm{ctf}$ & 496 & 1 & -2.58 & 1 & -6.53 & 1 & -2.82 & 223 & -0.15 & 1 & -6.28 \\
\hline $1 \mathrm{dtk}$ & 216 & 1 & -2.42 & 86 & -0.36 & 5 & -1.75 & 110 & 0.16 & 8 & -1.64 \\
\hline $1 \mathrm{fc} 2$ & 501 & 71 & -1.10 & 420 & 1.00 & 5 & -2.45 & - & - & 113 & -0.71 \\
\hline $1 \mathrm{igd}$ & 501 & 71 & -1.03 & 1 & -4.30 & 74 & -1.07 & 9 & -2.17 & 1 & -4.38 \\
\hline 1shf-A & 437 & 35 & -1.42 & 1 & -6.85 & 101 & -0.77 & 111 & -0.57 & 1 & -3.92 \\
\hline $2 \mathrm{cro}$ & 501 & 1 & -6.13 & 2 & -3.20 & 4 & -2.45 & - & - & 1 & -5.58 \\
\hline 2ovo & 348 & 37 & -1.27 & 1 & -8.53 & 26 & -1.36 & 12 & -2.44 & 1 & -6.60 \\
\hline 4pti & 344 & 3 & -2.54 & 1 & -6.28 & 14 & -1.78 & 245 & 0.81 & 1 & -3.48 \\
\hline Average & & 51.80 & -2.08 & 102.80 & -3.54 & 71.40 & -1.46 & 118.33 & -0.73 & 62.50 & -3.31 \\
\hline \multicolumn{12}{|c|}{ lattice_ssfit } \\
\hline 1 beo & 1998 & 61 & -2.06 & 1 & -19.51 & 1 & -3.43 & 41 & -1.76 & 1 & -13.89 \\
\hline $1 \mathrm{ctf}$ & 1999 & 1 & -4.03 & 1 & -10.97 & 1 & -3.48 & 1 & -10.64 & 1 & -13.75 \\
\hline $1 \mathrm{dkt}-\mathrm{A}$ & 1995 & 82 & -1.79 & 1 & -7.28 & 64 & -1.83 & 1 & -12.29 & 1 & -8.07 \\
\hline $1 \mathrm{fca}$ & 2001 & 72 & -1.96 & 1 & -8.82 & 65 & -1.84 & 1 & -11.71 & 1 & -7.74 \\
\hline $1 \mathrm{nkl}$ & 1995 & 1 & -3.86 & 1 & -4.44 & 2 & -3.28 & - & - & 1 & -6.36 \\
\hline $1 \mathrm{pgb}$ & 1997 & 65 & -1.98 & 1 & -15.56 & 45 & -1.99 & 1 & -28.85 & 1 & -16.66 \\
\hline $1 \mathrm{trl}-\mathrm{A}$ & 1999 & 347 & -0.97 & 1 & -7.49 & 527 & -0.63 & - & - & 1 & -7.58 \\
\hline $4 i c b$ & 1998 & 525 & -0.63 & 1 & -8.75 & 2 & -2.84 & 33 & -1.56 & 1 & -8.73 \\
\hline Average & & 144.25 & -2.16 & 1.00 & -10.35 & 88.38 & -2.42 & 13.00 & -11.14 & 1.00 & -10.35 \\
\hline \multicolumn{12}{|c|}{ Summary } \\
\hline Average & & 105.75 & -2.01 & 61.00 & -5.34 & 119.13 & -1.68 & 58.17 & -6.92 & 36.84 & -5.06 \\
\hline
\end{tabular}

a The decoy structures with broken backbone or missing fragments were removed from our test.

b The rank of native structure relative to decoy structures based on the calculated respective energies.

' The Z-score of native structure in the decoy structures.

${ }^{\mathrm{d}} E_{\text {beta }}$ is not evaluated due to no beta sheet in the protein. 
Table 2 Comparison of performance between NCACO-score and other potential functions on Decoys' $R^{\prime} U s$ sets.

\begin{tabular}{|c|c|c|c|c|c|c|c|c|c|c|c|c|c|c|c|c|c|c|}
\hline \multirow[b]{2}{*}{ ID } & \multirow[b]{2}{*}{ Size $^{a}$} & \multicolumn{2}{|c|}{ RAPDF } & \multicolumn{2}{|c|}{ Atomic KBP } & \multicolumn{2}{|c|}{ DFIRE-A } & \multicolumn{2}{|c|}{ DFIRE-B } & \multicolumn{3}{|c|}{ PC2CA } & \multicolumn{3}{|c|}{ DFMAC } & \multicolumn{3}{|c|}{ NCACO-score } \\
\hline & & $\operatorname{Rank}^{\mathrm{b}}$ & $\begin{array}{r}\text { Z- } \\
\text { score }^{c}\end{array}$ & Rank & $\begin{array}{r}\text { Z- } \\
\text { score }\end{array}$ & Rank & $\begin{array}{r}\text { Z- } \\
\text { score }\end{array}$ & Rank & $\begin{array}{r}\text { Z- } \\
\text { score }\end{array}$ & Rank & $\begin{array}{r}\text { Z- } \\
\text { score }\end{array}$ & C.C. & Rank & $\begin{array}{r}\text { Z- } \\
\text { score }\end{array}$ & C.C. & Rank & $\begin{array}{r}\text { Z- } \\
\text { score }\end{array}$ & C.C. \\
\hline \multicolumn{19}{|c|}{ 4state_reduced } \\
\hline $1 \mathrm{ctf}$ & 630 & 1 & -3.26 & 1 & -3.53 & 1 & -3.86 & 1 & -3.03 & 1 & -3.4 & 0.59 & 1 & -4.49 & 0.82 & 1 & -5.48 & 0.73 \\
\hline $1 \mathrm{r} 69$ & 676 & 1 & -3.49 & 1 & -3.76 & 1 & -4.23 & 1 & -2.95 & 1 & -4 & 0.62 & 1 & -3.34 & 0.82 & 1 & -4.47 & 0.6 \\
\hline $1 \mathrm{sn} 3$ & 660 & 1 & -3.26 & 1 & -3.5 & 1 & -3.79 & 1 & -3.4 & 1 & -3.6 & 0.36 & 1 & -3.14 & 0.55 & 1 & -4.73 & 0.46 \\
\hline $2 \mathrm{cro}$ & 673 & 1 & -2.93 & 1 & -2.91 & 1 & -3.29 & 2 & -2.74 & 1 & -3.2 & 0.69 & 1 & -3.17 & 0.82 & 1 & -4.36 & 0.59 \\
\hline 3icb & 654 & 1 & -2.22 & 1 & -2.41 & 4 & -2.28 & 24 & -1.68 & 1 & -2.9 & 0.76 & 1 & -2.01 & 0.86 & 1 & -3.33 & 0.78 \\
\hline $4 \mathrm{pti}$ & 686 & 1 & -3.12 & 1 & -3.47 & 1 & -3.62 & 1 & -3.15 & 1 & -3.1 & 0.40 & 1 & -4.3 & 0.52 & 1 & -6.1 & 0.35 \\
\hline $4 r \times n$ & 677 & 1 & -2.79 & 1 & -3.12 & 1 & -3.33 & 19 & -1.88 & 667 & 2.5 & 0.48 & 1 & -2.9 & 0.67 & 1 & -4.22 & 0.47 \\
\hline Average & & 1.00 & -3.01 & 1.00 & -3.24 & 1.43 & -3.49 & 7.00 & -2.69 & 96.14 & -2.53 & 0.56 & 1.00 & -3.34 & 0.72 & 1.00 & -4.67 & 0.58 \\
\hline \multicolumn{19}{|l|}{ fisa } \\
\hline $1 \mathrm{fc} 2$ & 501 & 497 & 2.74 & 413 & 1.05 & 254 & -0.23 & 1 & -2.76 & 1 & -6.6 & 0.11 & 399 & 0.77 & 0.50 & 461 & 1.68 & 0.4 \\
\hline $1 \mathrm{hdd}-\mathrm{C}$ & 501 & 17 & -2 & 25 & -1.78 & 1 & -4.5 & 1 & -6.76 & 1 & -8.4 & 0.24 & 1 & -4.81 & 0.39 & 21 & -1.55 & 0.33 \\
\hline $2 \mathrm{cro}$ & 501 & 14 & -1.93 & 24 & -1.64 & 1 & -6.33 & 1 & -7.84 & 1 & -7.3 & 0.17 & 1 & -4.19 & 0.28 & 3 & -2.43 & 0.26 \\
\hline $4 \mathrm{icb}$ & 500 & 1 & -3.89 & 6 & -2.46 & 1 & -6.91 & 1 & -8.47 & r & -9.3 & 0.23 & 1 & -5.1 & 0.25 & 1 & -4.43 & 0.23 \\
\hline Average & & 132.25 & -1.27 & 117.00 & -1.21 & 64.25 & -4.49 & 1.00 & -6.46 & 1.00 & -7.90 & 0.19 & 100.50 & -3.33 & 0.36 & 121.50 & -1.68 & 0.32 \\
\hline \multicolumn{19}{|c|}{ fisa_casp3 } \\
\hline 1 bg8-A & 1200 & 1 & -4.39 & 2 & -2.84 & 1 & -5.35 & 1 & -3.82 & 1 & -4.5 & 0.26 & 14 & -2.21 & 0.35 & 44 & -1.69 & 0.17 \\
\hline $1 \mathrm{bl0}$ & 972 & 1 & -3.19 & 215 & -0.76 & 1 & -4.5 & 3 & -2.27 & 1 & -3.1 & -0.09 & 8 & -2.17 & 0.30 & 3 & -2.46 & 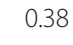 \\
\hline 1jwe & 1407 & 1 & -4.69 & 4 & -2.64 & 1 & -6.26 & 1 & -4.81 & 1 & -5.6 & 0.10 & 1 & -2.76 & 0.00 & 6 & -2.33 & -0.0 \\
\hline Average & & 1.00 & -4.09 & 73.67 & -2.08 & 1.00 & -5.37 & 1.67 & -3.63 & 1.00 & -4.40 & 0.09 & 7.67 & -2.38 & 0.22 & 17.67 & -2.16 & 0.15 \\
\hline \multicolumn{19}{|l|}{ Imds } \\
\hline $1 \mathrm{~b} 0 \mathrm{n}-\mathrm{B}$ & 498 & 359 & 0.45 & 74 & -1.03 & 430 & 1.17 & 261 & -0.03 & 1 & -3.3 & 0.05 & 1 & -2.82 & 0.07 & 1 & -2.56 & 0 \\
\hline $1 \mathrm{bba}$ & 501 & 501 & 11.11 & 500 & 3.51 & 501 & 16.28 & 501 & 21.38 & 501 & 21.4 & -0.23 & 501 & 4.38 & 0.18 & 497 & 2.02 & 0.2 \\
\hline $1 \mathrm{ctf}$ & 496 & 1 & -2.84 & 1 & -3.45 & 1 & -3.54 & 1 & -2.77 & 1 & -3.4 & 0.31 & 1 & -6.04 & 0.27 & 1 & -6.28 & 0.12 \\
\hline $1 \mathrm{dtk}$ & 216 & 116 & 0.08 & 31 & -1.16 & 1 & -2.62 & 5 & -2.46 & 2 & -2.5 & 0.21 & 70 & -0.38 & 0.04 & 8 & -1.64 & 0.16 \\
\hline $1 \mathrm{fc} 2$ & 501 & 501 & 7.75 & 501 & 8.86 & 501 & 5.72 & 441 & 1.22 & 53 & -1.3 & 0.17 & 501 & 2.94 & 0.08 & 113 & -0.71 & 0.0 \\
\hline $1 \mathrm{igd}$ & 501 & 1 & -4.21 & 1 & -4.16 & 1 & -5.16 & 1 & -4.69 & 1 & -4 & 0.10 & 1 & -7.21 & 0.25 & 1 & -4.38 & 0.15 \\
\hline 1shf-A & 437 & 1 & -5.15 & 2 & -2.83 & 1 & -6.68 & 1 & -5.44 & 1 & -5.3 & 0.11 & 1 & -4.28 & 0.06 & 1 & -3.92 & 0.02 \\
\hline $2 \mathrm{cro}$ & 501 & 416 & 0.96 & 175 & -0.4 & 1 & -4.7 & 1 & -4.5 & 1 & -7.7 & 0.13 & 1 & -3.04 & 0.07 & 1 & -5.58 & 0 \\
\hline $20 v 0$ & 348 & 4 & -2.76 & 1 & -2.86 & 1 & -3.21 & 27 & -1.48 & 1 & -3.2 & 0.11 & 1 & -2.73 & 0.16 & 1 & -6.6 & 0.1 \\
\hline $4 p t i$ & 344 & 157 & -0.2 & 13 & -1.75 & 1 & -3.96 & 1 & -3.47 & 1 & -3.5 & 0.02 & 3 & -2.57 & 0.10 & 1 & -3.48 & 0.17 \\
\hline Average & & 205.70 & 0.52 & 129.90 & -0.53 & 143.90 & -0.67 & 124.00 & -0.22 & 56.30 & -1.28 & 0.10 & 108.10 & -2.18 & 0.13 & 62.50 & -3.31 & 0.13 \\
\hline \multicolumn{19}{|c|}{ lattice_ssfit } \\
\hline 1 beo & 1998 & 1 & -9.79 & 1 & -9.47 & 1 & -12.09 & 1 & -7.95 & 1 & -5.6 & 0.08 & 1 & -8.37 & 0.04 & 1 & -13.89 & 0.0 \\
\hline $1 \mathrm{ctf}$ & 1999 & 1 & -6.99 & 1 & -7.2 & 1 & -10.05 & 1 & -6.89 & 1 & -6 & 0.03 & 1 & -10.22 & 0.01 & 1 & -13.75 & 0.10 \\
\hline $1 \mathrm{dkt}-\mathrm{A}$ & 1995 & 1 & -6.78 & 1 & -6.78 & 1 & -6.87 & 1 & -4.92 & 1 & -3.1 & -0.01 & 1 & -7.35 & -0.05 & 1 & -8.07 & 0.02 \\
\hline $1 \mathrm{fca}$ & 2001 & 1 & -5.57 & 1 & -3.36 & 1 & -7.18 & 1 & -5.3 & 1 & -4.7 & 0.04 & 1 & -9.31 & 0.01 & 1 & -7.74 & 0.0 \\
\hline $1 \mathrm{nkl}$ & 1995 & 1 & -8.33 & 1 & -8.16 & 1 & -9.29 & 1 & -5.83 & 1 & -4.1 & 0.01 & 1 & -5.18 & -0.09 & 1 & -6.36 & -0.14 \\
\hline $1 \mathrm{pgb}$ & 1997 & 1 & -8.42 & 1 & -6.86 & 1 & -11.87 & 1 & -9.64 & 1 & -4.7 & 0.04 & 1 & -13.65 & 0.14 & 1 & -16.66 & 0.13 \\
\hline $1 \mathrm{trl}-\mathrm{A}$ & 1999 & 1 & -4.84 & 1 & -5.58 & 1 & -6.32 & 1 & -3.73 & 1 & -3.6 & 0.02 & 1 & -4.09 & -0.02 & 1 & -7.58 & $0.0^{1}$ \\
\hline $4 \mathrm{icb}$ & 1998 & 1 & -6.68 & 1 & -5.65 & 1 & -7.81 & 1 & -4.25 & 1 & -4.4 & 0.00 & 1 & -5.98 & -0.02 & 1 & -8.73 & -0.02 \\
\hline Average & & 1.00 & -7.18 & 1.00 & -6.63 & 1.00 & -8.94 & 1.00 & -6.06 & 1.00 & -4.53 & 0.03 & 1.00 & -8.02 & 0.00 & 1.00 & -10.35 & 0.03 \\
\hline \multicolumn{19}{|l|}{ Summary } \\
\hline Average & & 81.38 & -2.83 & 62.59 & -2.88 & 53.66 & -4.27 & 40.81 & -3.32 & 39.09 & -3.48 & 0.19 & 47.53 & -4.05 & 0.26 & 36.84 & -5.06 & 0.2 \\
\hline
\end{tabular}

a The decoy structures with broken backbone or missing fragments were removed from our test.

${ }^{b}$ The rank of native structure relative to decoy structures based on the calculated respective energies.

c The Z-score of native structure in the decoy structures.

${ }^{d}$ The Pearson correlation coefficient between energy and CRMSD. 
For individual proteins, all the 6 functions showed similar trends with regard to their structure discriminating abilities. For example, they all discriminated 4state_reduced and lattice_ssfit sets with ease but were difficult to discriminate same proteins (e.g. $1 \mathrm{fc} 2$ in fisa set, $1 \mathrm{bba}$ and $1 \mathrm{fc} 2$ in lmds set). This phenomenon has been mentioned by other researchers before $[15,37]$. Zhou speculated that the failure of the scoring functions in 1 bba could be that it was an atypical small protein without a significant hydrophobic core [15].

\section{Application to de novo protein structure prediction}

Next, we sought to explore the performance of NCACO-score in structure modeling. Based on fragment assembly with three-residue fragments, we implemented a de novo structure prediction method that uses $\mathrm{NCACO}$-score to guide the fragment assembly process (see Methods). To promote the formation of correct beta sheet, and prevent the formation of wrong beta sheet, the weight of $E_{\text {beta }}$ was changed periodically according to a sine function during the fragment assembly procedure. In order to avoid the excessive collisions between atoms during the assembly process, a simple energy term for punishing collision was added to the NCACO-score. The procedure iterated three times, and the predicted structures from the previous iteration were used as the initial structures of the next iteration.

To compare our prediction method with Robetta, one of the best existing protein structure prediction servers, we tested 14 CASP8 hard targets, on which Robetta used template-free modeling method. For each target, 1000 structure models were generated in our method, then clustered and 5 top models were selected by a centroid-based clustering. Table 3 shows the accuracies of the top 1 models predicted by our method by comparing to the models that Robetta predicted in CASP8 which are available at the Robetta web site. As shown in the table, our method had average prediction accuracy of TM-score 0.290 , comparable to that by Robetta (0.287).

Although both our structure predictor and Robetta are based on fragment assembly, they have significantly different performance on modeling the structures. For example, for T0496-D2, our method correctly predicted its overall topology except for the coil part of its Nterminal $(\mathrm{TM}$-score $=0.463)$ (comparing the predicted and native structure of T0496-D2 in Figure 1), which is much better than Robetta prediction $(\mathrm{TM}$-score $=$ 0.291). For the 14 targets, the Pearson's correlation coefficient between TM-scores of our predicted structures and those of Robetta-predicted structures is very low (0.085). The complementarity between the two methods underscores the importance of our work on the development of NCACO-score for structure modeling.
Table 3 Comparison of performance between our method and Robetta on 14 CASP8 hard targets.

\begin{tabular}{lrrrr}
\hline & & & \multicolumn{2}{r}{ TM-score of Best Model } \\
\cline { 4 - 5 } ID & Length & $\begin{array}{r}\text { Secondary } \\
\text { Structure }\end{array}$ & $\begin{array}{r}\text { Robetta } \\
\text { (CASP8) }\end{array}$ & $\begin{array}{r}\text { Our } \\
\text { method }\end{array}$ \\
\hline T0397-D1 & 70 & 7beta & 0.25 & 0.277 \\
T0460 & 111 & 3alpha 5beta & 0.262 & 0.233 \\
T0465 & 157 & 5alpha 8beta & 0.243 & 0.309 \\
T0466 & 128 & 8beta & 0.326 & 0.172 \\
T0468 & 109 & 1alpha 7beta & 0.253 & 0.241 \\
T0476 & 108 & 4alpha 6beta & 0.279 & 0.241 \\
T0480 & 55 & 3beta & 0.208 & 0.217 \\
T0482 & 120 & 3alpha 5beta & 0.352 & 0.243 \\
T0495-D2 & 65 & 1alpha 2beta & 0.312 & 0.364 \\
T0496-D1 & 110 & 3alpha 6beta & 0.235 & 0.312 \\
T0496-D2 & 68 & 2alpha & 0.291 & 0.463 \\
T0510-D3 & 44 & 1alpha 3beta & 0.147 & 0.335 \\
T0513-D2 & 77 & 2alpha 4beta & 0.581 & 0.33 \\
T0514 & 145 & 2alpha 10beta & 0.283 & 0.322 \\
\hline Average & & & 0.287 & 0.29 \\
\hline
\end{tabular}

Moreover, our method guided by NCACO-score has a moderate computational cost. For the 14 targets, the average CPU time was $\sim 64.5 \mathrm{~h}$ on a $2.33 \mathrm{GHz}$ Intel Xeon processor.

\section{Discussion}

In this study, we have developed a knowledge-based scoring function named NCACO-score. NCACO-score integrates four different aspects of statistical structural features based on two coarse-grained models that only require coordinates of the heavy atoms of main-chain and pseudo side chain center of mass. We have demonstrated that NCACO-score can effectively discriminate

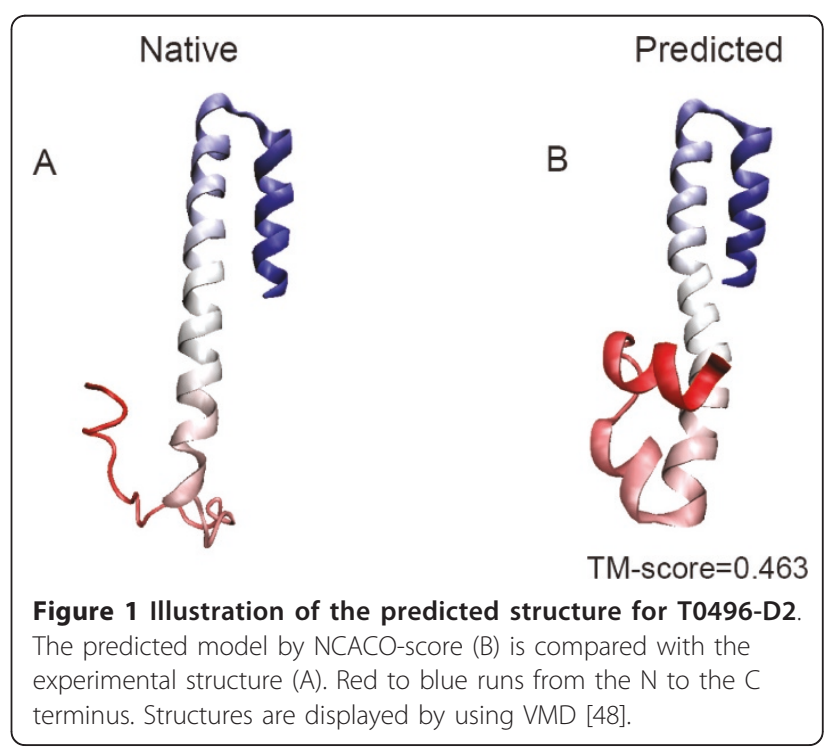


native structures from their decoys, with a performance comparable to or even better than other state-of-the-art coarse-grained or all-atom knowledge-based statistical scoring functions. Moreover, NCACO-score can be used to guide fragment assembly for fast structure prediction, which can achieve a comparable accuracy to Robetta, one of the best structure modellers of similar kind.

In developing knowledge-based scoring functions, orientation-dependent structural features have been widely used [11,21,23,38]. For examples, DFMAC uses an orientation-dependent potential term between two $\mathrm{C}_{\alpha}$ pseudo- $C_{\beta}$ vectors [21], and in OPUS- $C_{\alpha}$, the distancedependent pairwise energy term and hydrogen bonding energy term are also orientation-dependent [23]. These orientation-dependent potentials are sensitive to geometrical features, causing the energy to be truncated abruptly at the cutoff. Therefore, these orientationdependent potentials could have very rough energy landscape, which limit their applications to structural modeling. In NCACO-score, we exclude the orientationdependent potentials that are sensitive to geometrical features, and only the coordinates of the heavy atoms of main-chain and pseudo side chain center of mass are needed, greatly simplifying structure representation without need to scan the side chain conformations. Moreover, unlike the directional hydrogen-bonding potentials for capturing the interaction of beta sheet, which depend on critical geometrical constraints for atoms, the novel term we developed to capture beta sheet was based on the propensity of geometrical features between beta-strand parings. As the geometrical features are defined at $C_{\alpha}$ level in our model, the potential is less sensitive than typical directional hydrogen-bonding potential. We have shown that the novel beta sheet geometry propensity potential indeed had a good discrimination power for most proteins containing beta sheet.

Many potential terms were proposed and could be included in an effective knowledge-based scoring function. For example, Wu et al. added packing energy and three-body energy in their OPUS- $C_{\alpha}$ potential [23], Makino and Itoh added the potential of $\omega$ dihedral angle in their DFMAC potential [21], and Fogolari et.al. added pseudo bond/angle/dihedral potentials in their PC2CA potential [22]. These potential terms are proved effective more or less in structure discrimination, which will be attempted in NCACO-score in our future work. The inclusion of more potential terms could improve the performance of structure discrimination, but this could render the energy landscape too rough and cause overfitting [39]. In developing NCACO-score, we have only considered four terms that reflect different aspects of protein folding principles. Indeed, integration of these four terms improves the performance of NCACO-score, although $E_{t r p}$ makes a dominant contribution.

\section{Conclusions}

NCACO-score is a knowledge-based scoring function that integrates four statistical structural features to effectively discriminate native structures from their decoys. Successful application of NCACO-score to structure modeling shows that NCACO-score could be a useful tool for structure modeling.

\section{Methods}

\section{Nonhomologous structure database}

6997 protein structures (less than 25\% homology) with a resolution $<3.0 \AA$ and $\mathrm{R}$-factor $<1.0$ are obtained from PISCES server (May 20, 2010) [40], and are used to derive statistical potentials. For determining the distance cutoff of contact for any two types of atom, we need to get the minimal distance between any atom pair in protein structures (the detail of contact definition is below). So, a high-resolution structure database is used, including 2069 protein structures (less than 25\% homology) with a resolution $<1.6 \AA$ and $\mathrm{R}$-factor $<0.25$ from PISCES server (May 20, 2010).

\section{Knowledge-based scoring function: NCACO-score 1. Coarse-grained structure representations}

To calculate $E_{c o n}$ and $E_{t r p}$, NCACO-score uses a coarsegrained model of five beads, which includes 5 atoms (N $\mathrm{C} \alpha \mathrm{C} \mathrm{O}$ in backbone, and pseudo side chain center of mass) for representing a residue (Figure 2). For the $\chi_{1} /$ $\chi_{2}$ torsion angles of side chain were dependent on its backbone $\phi / \psi$ torsion angles [41], the pseudo side chain center of mass was determined by its backbone $\phi / \psi$ torsion angles in our model, which is described as follows:

First, the side chain centers of mass (SCM) excluding $\mathrm{H}$ atoms for all residues of 6997 proteins are calculated. The $\phi / \psi$ torsion angles of backbone, the pseudo bond length $(\mathrm{C} \alpha-\mathrm{SCM})$, the pseudo bond angle (N-C $\alpha-\mathrm{SCM})$, and the pseudo bond dihedral (N-C-C $\alpha-\mathrm{SCM})$ are calculated for each residues. Second, the $\phi / \psi$ torsion angles (ranging from $-180^{\circ}$ to $180^{\circ}$ ) are divided into 36 bins with a width of $10^{\circ}$. The values of pseudo bond length,

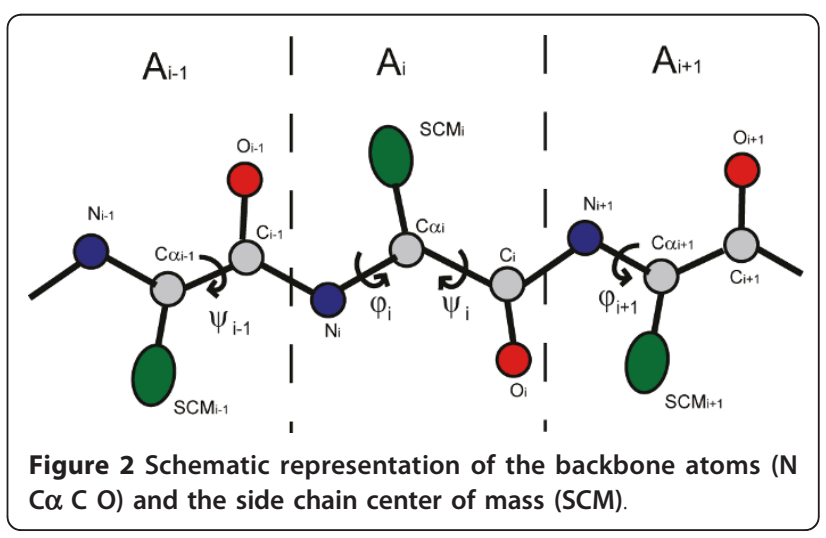


angle, and dihedral of the 19 amino acids (except GLY) according to each of the $36 * 36$ bins are averaged on the 6997 proteins. Thus, a table containing the average pseudo bond length/angle/dihedral for each of all $19 * 36 * 36$ pseudo SCM is obtained. Finally, given the four backbone atoms $(\mathrm{N} \mathrm{C} \alpha \mathrm{C} \mathrm{O})$ coordinates of a structure, the pseudo SCM information (bond length/ angle/dihedral) for each residue is extracted from the table according to its amino acid type and $\phi / \psi$ torsion angles of backbone. The coordinate of pseudo SCM atom of each residue can be calculated by its backbone atoms ( $\mathrm{N} \mathrm{C \alpha} \mathrm{C} \mathrm{O)} \mathrm{coordinates} \mathrm{and} \mathrm{its} \mathrm{pseudo} \mathrm{SCM}$ information through coordinates transformation.

To calculate $E_{\text {sol }}$ and $E_{\text {beta }}$, we use residue-level coarse-grained model, in which a residue is represented as its $\mathrm{C} \alpha$ atom. Therefore, only the coordinates of $\mathrm{C} \alpha$ atoms are needed.

\section{Individual energy terms}

The pairwise atom-atom contact potential According to our five-bead coarse-grained model, there are four atoms ( $\mathrm{N} \mathrm{C} \alpha \mathrm{C} \mathrm{O}$ ) for GLY and five atoms ( $\mathrm{N} \mathrm{Ca} \mathrm{C} \mathrm{O}$ SCM) for the other 19 amino acids, resulting in all 99 atom types. Two atoms $\mathrm{A}$ and $\mathrm{B}$ are defined to be in contact if their distance $d_{A B}$ is between $r_{A B}$ and $\lambda^{*} r_{A B}$, where $r_{A B}$ is the statistical shortest contact distance between atoms A and B observed in the 2069 high-resolution protein structures $\left(r_{A B}>2.2 \AA\right.$ to exclude the situation that two atoms could be covalently linked). $\lambda$ $=1.9$ is used in our model. Then, for each pair of atoms $\mathrm{A}$ and $\mathrm{B}$, the number of contacts $\left(N_{A B}\right)$ and number of non-contacts $\tilde{N}_{A B}$ are counted in the 6997 proteins. By following the method of Shakhnovich lab [11], the contact potential uses the form of $\mu$-potential defined as:

$$
\begin{aligned}
& E_{A B}= \begin{cases}0 \quad \text { if } N_{A B}<10 \text { and } \tilde{N}_{A B}<10 \\
\frac{-\mu N_{A B}+(1-\mu) \tilde{N}_{A B}}{\mu N_{A B}+(1-\mu) \tilde{N}_{A B}} & \text { else }\end{cases} \\
& E_{\text {con }}=\sum_{i<j} E_{A_{i} A_{j}}
\end{aligned}
$$

The value of $\mu=0.987$ is chosen to make the net interaction zero. $E_{c o n}$ for a protein is summed over the energies of all possible atom pairs in the protein.

The sequence-dependent local conformational potential The Ramachandran map suggests that the distribution of $\phi / \psi$ torsion angles for a polypeptide is limited, and the distributions of $\phi / \psi$ torsion angles are different for different amino acids. We consider the local conformation potential based on three-residue fragment. Figure 2 shows a triplet fragment consisting of $\mathrm{A}_{i-1}, \mathrm{~A}_{i}$, $\mathrm{A}_{i+1}$, where $\mathrm{A}_{i}$ is the amino acid type of the $i$-th residue.
The four dihedrals $\psi_{i-1} \phi_{i} \psi_{i} \phi_{i+1}$ (range from $-180^{\circ}$ to $180^{\circ}$ ) are used to express the local conformation potential of a triplet fragment. Each dihedral is divided into 12 bins with bin width $30^{\circ}$. By following the method of Shakhnovich lab [11], the sequence-dependent local conformation potential is also expressed as a $\mu$-potential:

$$
\begin{aligned}
& E_{A_{i-1} A_{i} A_{i+1}}=\left\{\begin{array}{l}
0 \quad \text { if } N_{j}<10 \text { and } \tilde{N}_{j}<10 \\
\frac{-\mu N_{j}+(1-\mu) \tilde{N}_{j}}{\mu N_{j}+(1-\mu) \tilde{N}_{j}} \quad \text { else }
\end{array}\right. \\
& E_{\text {trp }}=\sum_{i} E_{A_{i-1} A_{i} A_{i+1}}
\end{aligned}
$$

where $N_{j}$ and $\tilde{N}_{j}$ are the number of occurrence of the triplet $\mathrm{A}_{i-1} \mathrm{~A}_{i} \mathrm{~A}_{i+1}$ in the $j$-th bin and the total number of occurrence of the triplet $\mathrm{A}_{i-1} \mathrm{~A}_{i} \mathrm{~A}_{i+1}$ subtracted by $N_{j}$ in the 6997 proteins, respectively. The value of $\mu=$ 0.994 is chosen to make the net interaction zero. $E_{\text {trp }}$ for a protein is summed over the energies of all overlap triplet fragments in the protein.

The solvation potential The solvation potential is derived at residue level by considering $\mathrm{C} \alpha$ atom only, which is similar to the SURR term used in DFMAC potential [21]. It is described as follows:

$$
\begin{aligned}
& E_{a k}= \begin{cases}4.0 & \text { if } N_{a k}=0 \text { or } \tilde{N}_{a k}=0 \\
0 & \text { else if } N_{a k}<10 \text { and } \tilde{N}_{a k}<10 \\
-\log \frac{N_{a k}}{\tilde{N}_{a k}} & \text { else }\end{cases} \\
& \tilde{N}_{a k}=\frac{N_{a} * N_{k}}{N_{\text {total }}} \\
& E_{\text {sol }}=\sum_{i} E_{A_{i}}
\end{aligned}
$$

where $\mathrm{A}_{i}$ is the type of the $i$-th residue, and $E_{a k}$ is the potential for amino acid $a$ with $k$ contact residues. $N_{a k}$ is the number of observations for amino acid $a$ with $k$ contact residues in the 6997 proteins, and $\tilde{N}_{a k}$ (the expectation of $N_{a k}$ ) is estimated by equation 7 . In the equation, $N_{a}$ is the total number of occurrence of amino acid $a, N_{k}$ is the total number of residues with $k$ contact residues, and $N_{\text {total }}$ is the total number of residues in the database. In this procedure two residues are regarded to be in contact if the distance between their $\mathrm{C} \alpha$ atoms is less than $12.5 \AA$. $E_{\text {sol }}$ for a protein is summed over the energies of all residues in the protein.

The beta sheet geometry propensity potential Hydrogen bond energy plays an important role in the formation of beta sheet. Thus far, most methods have 
attempted to calculate hydrogen-bond donor and acceptor explicitly to capture beta sheet $[11,23,38]$, which are computationally intensive and cause the non-smooth truncation of energy at the geometric boundaries. To overcome the shortcoming, we used the geometrical features of beta sheet to guide the formation of beta sheet correctly based on a $\mathrm{C} \alpha$ level coarse-grained model. As shown in Figure 3A, both parallel and anti-parallel beta sheet have similar backbone geometrical feature at $\mathrm{C} \alpha$ level. Our model considers the pairing of three-residue fragments. First, we determine whether a pair of triplet fragments has a tendency to form beta sheet according to a simple criteria: all the three distances $d_{C A_{i-1} C A_{j-1}} d_{C A_{i} C A_{j}}$ and $d_{C A_{i+1} C A_{j+1}}$ (see Figure $3 \mathrm{~B}$ ), are required to be less than a cutoff $d$ for parallel beta sheet. While for anti-parallel beta sheet, the three distances are $d_{C A_{i-1} C A_{j+1}} d_{C A_{i} C A_{j}}$ and $d_{C A_{i+1} C A_{j-1}}$. The tendencies of both parallel and anti-parallel patterns are considered. If the both patterns are satisfied the criteria, we compare the average value of the three distances and choose the pattern that has a smaller average distance. Second, the four features $\alpha 1 \alpha 2 \beta$ and $\gamma$ (illustrated in Figure $3 \mathrm{~B}$ ) of a fragment pair are calculated for all fragment pairs that have tendencies to form beta sheet in the 6997 proteins. Then the four features are binned according to their range. The statistical results point that $\alpha 1$ and $\alpha 2$ fall into the range between $70^{\circ}$ to $180^{\circ}$ (varying slightly on different parameter $d$ ), which are separated into four bins: $0^{\circ} \sim 90^{\circ}, 90^{\circ} \sim 120^{\circ}, 120^{\circ} \sim 150^{\circ}$, and $150^{\circ} \sim 180^{\circ} ; \beta$ and $\gamma$ fall into the range between $0^{\circ}$ to $180^{\circ}$, which are separated into six bins: $0^{\circ} \sim 30^{\circ}, 30^{\circ} \sim 60^{\circ}$, $60^{\circ} \sim 90^{\circ}, 90^{\circ} \sim 120^{\circ}, 120^{\circ} \sim 150^{\circ}$, and $150^{\circ} \sim 180^{\circ}$. In total, there are $4 \times 4 \times 6 \times 6=576$ bins for a fragment pair. Based on the Boltzmann law [42], the potential is derived as follows:

$$
\begin{aligned}
& E_{\alpha 1 \alpha 2 \beta \lambda}= \begin{cases}10.0 & \text { if } N_{\alpha 1 \alpha 2 \beta \lambda}=0 \\
-\log \frac{N_{\alpha 1 \alpha 2 \beta \lambda}}{\tilde{N}} & \text { else }\end{cases} \\
& \tilde{N}=\frac{\sum_{i} N_{i}}{4 \times 4 \times 6 \times 6} \\
& E_{\text {beta }}=\sum_{i \in \text { fragment pairs }} E_{i}
\end{aligned}
$$

where $N_{\alpha 1 \alpha 2 \beta \lambda}$ is the number of observations of all fragment pair in each bin of $\alpha 1 \alpha 2 \beta \gamma$ in the database. $\tilde{N}$ is the average number of observations for each bin. For parameter $d$, we tested its range from $5.0 \AA$ to $6.4 \AA$ and finally set it to $5.6 \AA$ which gives a better

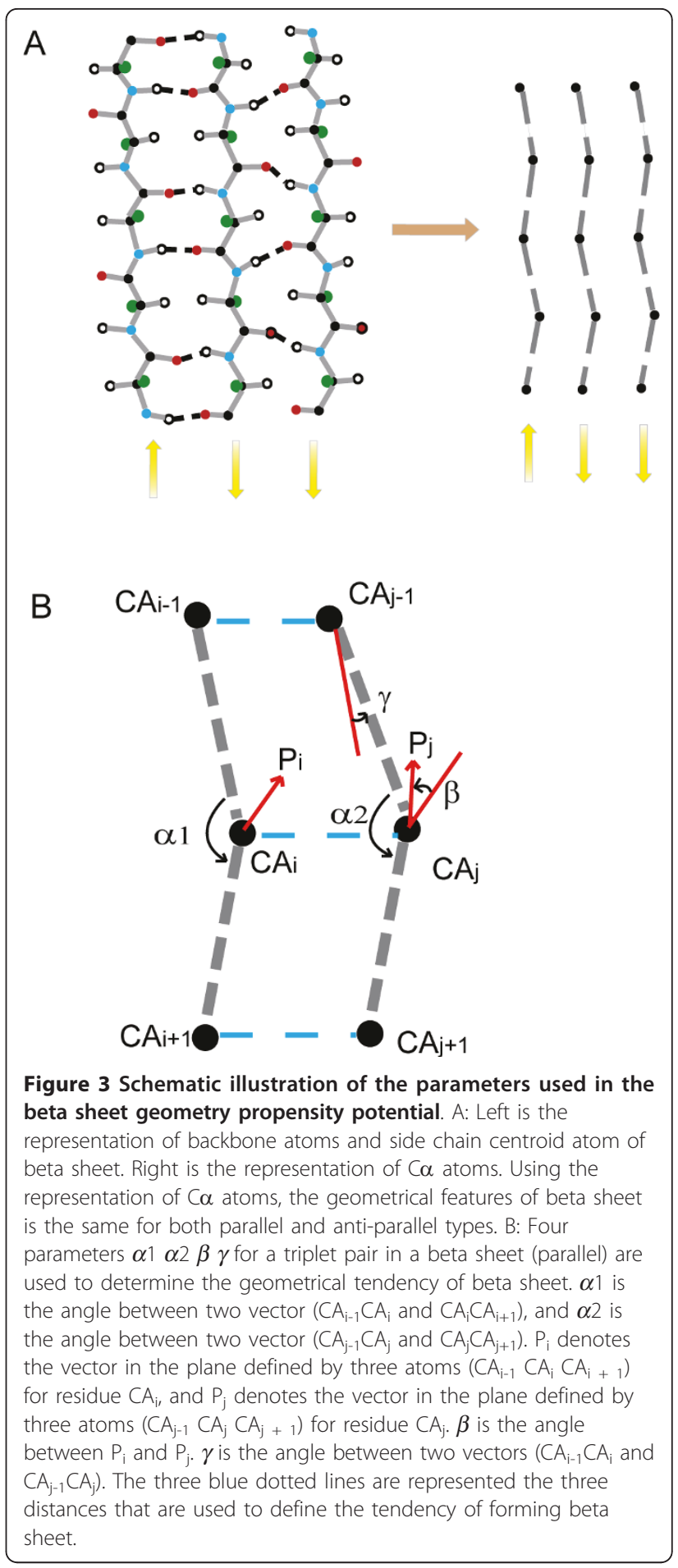

optimization result. $5.6 \AA$ is close to the average distance between backbones of beta sheet in the structure database. $E_{\text {beta }}$ for a protein is summed over the energies of all triplet fragment pairs that have tendencies to form beta sheet in the protein. 


\section{Integration of the four terms into NCACO-score}

The above four terms are linearly combined to form NCACO-score:

$$
E_{\text {tot }}=E_{c o n}+a \times E_{\text {trp }}+b \times E_{s o l}+c \times E_{b e t a}
$$

where a, b, c are the relative weights of $E_{t r p}, E_{\text {sol }}$ and $E_{\text {beta }}$ respectively, which are parameterized on 32 Decoys'R'Us sets (see below).

\section{Optimization of weights and parameters}

Weights of the equation 12 and parameters in the single potentials described above are optimized on 32 proteins of Decoys'R'Us sets (described above). The cost function for optimization is:

$$
F=\bar{N}_{n}+8.0 \bar{Z}
$$

where $\bar{N}_{n}$ is the average rank of native structure and $\bar{Z}$ is the average $Z$-score for the proteins used for training. The optimization goal is to minimize the function value. After optimization on all 32 proteins, the NCACO-score is

$$
E_{\text {tot }}=E_{c o n}+5.4 \times E_{\text {trp }}+12.3 \times E_{\text {sol }}+3.1 \times E_{\text {beta }}
$$

\section{Performance assessment}

Two commonly used indices are used for quality measures: $\mathrm{Rn}$, the rank of native structure relative to decoy structures based on the calculated energy; Z-score, a quantitative measure of energy bias for the native structure against decoy structures, which is defined as:

$$
z=\frac{E_{\text {tot }}^{\text {native }}-\bar{E}_{t o t}}{\sigma\left(E_{t o t}\right)},
$$

$E_{\text {tot }}^{\text {native }}$ and $E_{\text {tot }}$ are the energies of the native and decoy structures, respectively, $\bar{E}_{t o t}$ and $\sigma\left(E_{t o t}\right)$ are the average and standard deviation of energy of all decoys, respectively.

\section{Development a de novo structure prediction method using fragment assembly guided by NCACO-score}

We applied NCACO-score to structure modeling based on fragment assembly similar to that was used in Rosetta [33].

1. Fragment templates library In order to compare our prediction results with Robetta results in CASP8 fairly, the proteins in the database consisting of 6997 proteins that have more than $30 \%$ identity to the predicted target are excluded, which guarantees there are no homologous proteins for the predicted target. The list of fragment templates for the target proteins are generated as follows:

First, we obtained the secondary structure information for both target proteins and the template structures from the nonhomologous structure database. The secondary structures of target proteins are predicted using PSIPRED [43]. The secondary structures of the proteins in the nonhomologous structure database are identified using DSSP [44]: H, G, I for alpha helix, E for betastrand, and the others for coil. Second, sequence profiles for each target sequence and each sequence of the structure database are constructed by three rounds of PSI-BLAST [45] with an e-value cutoff of 0.001. Finally, sequences of the target protein and the structure database are cut to overlapping triplet fragments. All triplet fragments of the structure database are scored with each triplet fragment of the target sequence using the following scoring function:

$$
\begin{aligned}
& \begin{aligned}
S(\text { query, template }) & =S_{\text {profile }}(\text { query, template }) \\
& +S_{s s}(\text { query, template })
\end{aligned} \\
& S_{\text {profile }}(\text { query, template })=\sum_{i=1}^{3} \sum_{j=1}^{20} F_{\text {query }}(i, j) P_{\text {template }}(i, j) \\
& S_{s s}(\text { query, template })=\sum_{i=1}^{3} S\left(q_{i}, t_{i}\right)
\end{aligned}
$$

$$
S\left(q_{i}, t_{i}\right)=\left\{\begin{array}{l}
1 \quad \text { if } q_{i}=t_{i} \\
0 \quad \text { else if } q_{i} \text { or } t_{i} \text { is 'coil' } \\
-1 \quad \text { else }
\end{array}\right.
$$

Where $F_{\text {query }}(i, j)$ and $P_{\text {template }}(i, j)$ are the frequency matrix of the query triplet and log-odds matrix of the template triplet for amino acid $j$ at the $i$-th position, respectively. $q_{i}$ and $t_{i}$ are the secondary structure of query triplet and template triplet at the $i$-th position, respectively. After ranking, for each query triplet of the target protein, 25 triplets of top scores from the structure database are selected. The $\phi / \psi / \omega$ torsion angles of template triplets are obtained as the fragment templates library.

2. NCACO-score-guided structure modeling process $\mathrm{NCACO}$-score can be used to guide fragment assembly for protein structure prediction, which is detailed as follows:

Step 1. An extended backbone structure is initialized for the target sequence, in which all $\phi / \psi / \omega$ torsion angles are $180^{\circ}$.

Step 2. Template triplets in the fragment templates library are randomly selected to replace the value of $\phi / \psi / \omega$ torsion angles of the target sequence from $\mathrm{N}$ term to $\mathrm{C}$-term. Thus a random initial backbone structure is constructed, whose $\phi / \psi / \omega$ torsion angles of backbone are all from the fragment templates library.

Step 3. A triplet in the target sequence is randomly selected as the target fragment, and is replaced by randomly selected template triplet. The energies of the structures are evaluated by NCACO-score with atom clash punishment and promotion of beta sheet (described below). Whether to reject or accept a 
replacement is based on the metropolis acceptance criteria: a replacement with lower energy is accepted, and a replacement with higher energy is occasionally accepted. The details are described below:

$$
\begin{aligned}
& \text { if } e^{-\frac{E_{\text {new }}-E_{\text {old }}}{k b \times \text { factor } \times T}}<r \text {, reject } \\
& \text { else, }
\end{aligned}
$$

where $\mathrm{r}$ is a random float number between 0 and 1 . 35,000 replacements are implemented with a simulated annealing protocol. The temperature $(\mathrm{T})$ is gradually decreased from $2000 \mathrm{~K}$ to $300 \mathrm{~K}$. The initial factor is 1.0 . In order to avoid local optima, if the times of continuous rejection $(\mathrm{Nr})$ is more than 200 , factor $=1.0+(\mathrm{Nr}-$ 200)/80.0; and if the factor is more than 11.0, the simulated annealing process is stopped.

Step4. The step2 and step3 are repeated for 1000 times to generate 1000 predicted structures for the target sequence.

Step5. Model selection. The 1000 predicted structures are clustered using a centroid-based clustering process by following the algorithm of SPICKER [46] with adaptation. The top five biggest clusters are selected as top five predicted structures. Different from SPICKER that uses RMSD to assess structural similarity for clustering, our implemented centroid-based clustering process uses TM-score, which is more sensitive than RMSD [47]. The initial TM-score cutoff is set to 0.7, the TM-score cutoff for clustering should be between 0.3 and 0.9 , and the biggest cluster should includes $20 \% \sim 70 \%$ of the total number of structures.

The above procedure iterated for three times. In the second run, the top five predicted structures in the first run are used as initial structures. For each of the initial structures, the assembly procedure is implemented for 200 times, and it results in 200 predicted structures. The factor of metropolis acceptance criteria is different from that for the first run: if the times of continuous rejections $\mathrm{Nr}>200$, factor $=1.0+(\mathrm{Nr}-200) / 160.0$; and if factor $<6.0$, the simulated annealing process is stopped. Similarly, the top five structures predicted in the second run are used as initial structures for the third run, and 200 times assembly procedures result in 200 predicted structures for each initial structure. In the third run, if the times of continuous rejection $\mathrm{Nr}>200$, factor $=1.0+(\mathrm{Nr}-200) /$ 320.0 ; and if the factor $<3.5$, the simulated annealing process is stopped. Finally, the structure with the lowest energy among the top five predicted structures from the third run is obtained as the best predicted model.

\section{Atom clash punishment and promotion of beta} sheet During the process of fragment assembly, atom clash happens frequently. Atoms A and B are regarded to have clash with each other if the distance $d_{A B}$ between them is less than the statistical shortest contact distance between them $r_{A B}$ (as described in the pairwise atom-atom contact potential part). Two measures are used to punish the atom clash. One is based on the number of all backbone atom pairs having clash $\left(N_{b b_{-} \text {clash }}\right)$. If $N_{b b_{-} \text {clash }}>N_{\text {seq }} / 3.0$ (where $N_{\text {seq }}$ is the sequence length of the target protein), the structure is rejected. The other is to add a clash punishment term $E_{\text {clash }}=N_{\text {clash }}$ to the NCACO-score. $N_{\text {clash }}$ is the total number of atom pairs in clash including the backbone atoms and the pseudo side chain center atoms.

As a long-range interaction, beta sheet is hard to form correctly during the fragment assembly process. To promote the correct formation of beta sheet, the weight of $E_{\text {beta }}$ varies by multiplying a periodic factor $10.0 * \sin \left(\frac{T n}{1000} * \pi\right)$, where $\mathrm{Tn}$ is the step of random fragment replacement. Thus, the weight is increased during the first 500 steps. Then the weight is decreased and increased alternately.

\section{Additional material}

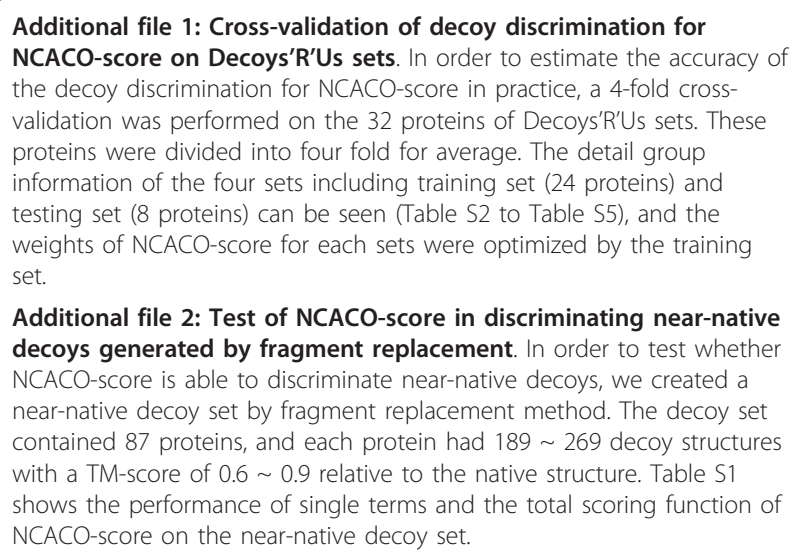

Additional file 2: Test of NCACO-score in discriminating near-native decoys generated by fragment replacement. In order to test whether NCACO-score is able to discriminate near-native decoys, we created a near-native decoy set by fragment replacement method. The decoy set contained 87 proteins, and each protein had $189 \sim 269$ decoy structures with a TM-score of $0.6 \sim 0.9$ relative to the native structure. Table S1 shows the performance of single terms and the total scoring function of NCACO-score on the near-native decoy set.

\section{Acknowledgements}

We thank the members of Jiang lab for discussions. This study was supported by the Bai Ren Project of Chinese Academy of Sciences and grants from the Chinese Ministry of Science and Technology, the National Science and Technology Key Project (2008ZX10004-013) and Project 973 (2009CB918503) to TJ. The results described in this paper are obtained on the Deepcomp7000 of Supercomputing Center, Computer Network Information Center of Chinese Academy of Sciences. The authors would like to thank Mr. Yoshihide Makino for providing the data of DFMAC potential. The source codes and parameter files of NCACO-score can be downloaded free for academic use from http://jianglab.ibp.ac.cn/lims/ncaco/ncaco.html.

\section{Author details}

${ }^{1}$ National Laboratory of Biomacromolecules, Institute of Biophysics, Chinese Academy of Sciences, Beijing 100101, China. ${ }^{2}$ Graduate School of the Chinese Academy of Sciences, Beijing 100080, China.

\section{Authors' contributions}

$L T, A W$ and TJ designed the research. LT and AW carried out the study. LT and TJ drafted the manuscript. All authors contributed to the discussion and 
helped to draft the manuscript. All authors have read and approved the final manuscript.

Received: 20 January 2011 Accepted: 26 May 2011

Published: 26 May 2011

\section{References}

1. Cheatham TE, Young MA: Molecular dynamics simulation of nucleic acids: successes, limitations, and promise. Biopolymers 2000, 56(4):232-256.

2. Mackerell AD, Wiorkiewiczkuczera J, Karplus M: An All-Atom Empirical Energy Function for the Simulation of Nucleic-Acids. J Am Chem Soc 1995, 117(48):11946-11975.

3. Boas FE, Harbury PB: Potential energy functions for protein design. Curr Opin Struc Biol 2007, 17(2):199-204.

4. Arab S, Sadeghi M, Eslahchi C, Pezeshk H, Sheari A: A pairwise residue contact area-based mean force potential for discrimination of native protein structure. Bmc Bioinformatics 2010, 11:16.

5. Skolnick J: In quest of an empirical potential for protein structure prediction. Curr Opin Struc Biol 2006, 16(2):166-171.

6. Dill KA, Ozkan SB, Shell MS, Weikl TR: The protein folding problem. Annu Rev Biophys 2008, 37:289-316.

7. Poole AM, Ranganathan R: Knowledge-based potentials in protein design. Curr Opin Struc Biol 2006, 16(4):508-513.

8. Sippl MJ: Knowledge-Based Potentials for Proteins. Curr Opin Struc Biol 1995, 5(2):229-235

9. Macarthur MW, Driscoll PC, Thornton JM: Nmr and Crystallography Complementary Approaches to Structure Determination. Trends Biotechnol 1994, 12(5):149-153.

10. Zhang C, Liu S, Zhu Q, Zhou Y: A knowledge-based energy function for protein-ligand, protein-protein, and protein-DNA complexes. J Med Chem 2005, 48(7):2325-2335

11. Yang JS, Chen WW, Skolnick J, Shakhnovich El: All-atom ab initio folding of a diverse set of proteins. Structure 2007, 15(1):53-63.

12. Potapov V, Cohen M, Inbar Y, Schreiber G: Protein structure modelling and evaluation based on a 4-distance description of side-chain interactions. Bmc Bioinformatics 2010, 11:374.

13. Lu H, Skolnick J: A distance-dependent atomic knowledge-based potential for improved protein structure selection. Proteins 2001, 44(3):223-232

14. Lu MY, Dousis AD, Ma JP: OPUS-PSP: An orientation-dependent statistical all-atom potential derived from side-chain packing. J Mol Biol 2008, 376(1):288-301.

15. Zhou H, Zhou Y: Distance-scaled, finite ideal-gas reference state improves structure-derived potentials of mean force for structure selection and stability prediction. Protein Sci 2002, 11(11):2714-2726.

16. Melo F, Feytmans E: Novel knowledge-based mean force potential at atomic level. J Mol Biol 1997, 267(1):207-222.

17. Shen MY, Sali A: Statistical potential for assessment and prediction of protein structures. Protein Sci 2006, 15(11):2507-2524.

18. McConkey BJ, Sobolev V, Edelman M: Discrimination of native protein structures using atom-atom contact scoring. P Natl Acad Sci USA 2003 100(6):3215-3220.

19. Samudrala R, Moult J: An all-atom distance-dependent conditional probability discriminatory function for protein structure prediction. $J \mathrm{Mol}$ Biol 1998, 275(5):895-916

20. Helles G: A comparative study of the reported performance of ab initio protein structure prediction algorithms. Journal of the Royal Society Interface 2008, 5(21):387-396.

21. Makino $Y$, Itoh N: A knowledge-based structure-discriminating function that requires only main-chain atom coordinates. Bmc Struct Biol 2008, 8:46.

22. Fogolari F, Pieri L, Dovier A, Bortolussi L, Giugliarelli G, Corazza A Esposito G, Viglino P: Scoring predictive models using a reduced representation of proteins: model and energy definition. BmC Struct Biol 2007, 7:15.

23. Wu YH, LU MY, Chen MZ, Li JL, Ma JP: OPUS-Ca: A knowledge-based potential function requiring only C alpha positions. Protein Sci 2007, 16(7):1449-1463

24. Go JF, Li HL, Jiang HL, Wang XC: A simple C-alpha-SC potential with higher accuracy for protein fold recognition. Biochem Bioph Res Co 2009, 379(2):610-615.
25. Miyazawa S, Jernigan RL: Residue-residue potentials with a favorable contact pair term and an unfavorable high packing density term, for simulation and threading. J Mol Biol 1996, 256(3):623-644.

26. Zhang J, Chen R, Liang J: Empirical potential function for simplified protein models: Combining contact and local sequence-structure descriptors. Proteins-Structure Function and Bioinformatics 2006, 63(4):949-960.

27. Fitzgerald JE, Jha AK, Colubri A, Sosnick TR, Freed KF: Reduced C-beta statistical potentials can outperform all-atom potentials in decoy identification. Protein Sci 2007, 16(10):2123-2139.

28. Rajgaria R, McAllister SR, Floudas CA: A novel high resolution C-alpha-Calpha distance dependent force field based on a high quality decoy set. Proteins-Structure Function and Bioinformatics 2006, 65(3):726-741.

29. Hinds DA, Levitt M: A Lattice Model for Protein-Structure Prediction at Low Resolution. P Natl Acad Sci USA 1992, 89(7):2536-2540.

30. Zhang C, Liu S, Zhou HY, Zhou YQ: An accurate, residue-level, pair potential of mean force for folding and binding based on the distancescaled, ideal-gas reference state. Protein Sci 2004, 13(2):400-411.

31. Bahar I, Jernigan RL: Inter-residue potentials in globular proteins and the dominance of highly specific hydrophilic interactions at close separation. J Mol Biol 1997, 266(1):195-214.

32. Zhang C, Kim SH: Environment-dependent residue contact energies for proteins. P Natl Acad Sci USA 2000, 97(6):2550-2555.

33. Rohl CA, Strauss CEM, Misura KMS, Baker D: Protein structure prediction using rosetta. Method Enzymol 2004, 383:66-93.

34. Samudrala R, Levitt M: Decoys ' $R$ ' Us: A database of incorrect conformations to improve protein structure prediction. Protein Sci 2000 9(7):1399-1401.

35. Kim DE, Chivian D, Baker D: Protein structure prediction and analysis using the Robetta server. Nucleic Acids Res 2004, 32:W526-W531.

36. Park B, Levitt M: Energy functions that discriminate $X$-ray and near-native folds from well-constructed decoys. J Mol Biol 1996, 258(2):367-392.

37. Tobi D, Elber R: Distance-dependent, pair potential for protein folding: Results from linear optimization. Proteins-Structure Function and Genetics 2000, 41(1):40-46.

38. Kortemme T, Morozov AV, Baker D: An orientation-dependent hydrogen bonding potential improves prediction of specificity and structure for proteins and protein-protein complexes. J Mol Biol 2003, 326(4):1239-1259.

39. Cohen M, Potapov V, Schreiber G: Four Distances between Pairs of Amino Acids Provide a Precise Description of their Interaction. PLoS Comp Biol 2009, 5(8):e1000470.

40. Wang GL, Dunbrack RL: PISCES: a protein sequence culling server. Bioinformatics 2003, 19(12):1589-1591.

41. Bower MJ, Cohen FE, Dunbrack RL: Prediction of protein side-chain rotamers from a backbone-dependent rotamer library: A new homology modeling tool. J Mol Biol 1997, 267(5):1268-1282.

42. Sippl MJ: Calculation of Conformational Ensembles from Potentials of Mean Force - an Approach to the Knowledge-Based Prediction of Local Structures in Globular-Proteins. J Mol Biol 1990, 213(4):859-883.

43. Jones DT: Protein secondary structure prediction based on positionspecific scoring matrices. J Mol Biol 1999, 292(2):195-202.

44. Kabsch W, Sander C: Dictionary of protein secondary structure: pattern recognition of hydrogen-bonded and geometrical features. Biopolymers 1983, 22(12):2577-2637.

45. Altschul SF, Madden TL, Schaffer AA, Zhang JH, Zhang Z, Miller W, Lipman DJ: Gapped BLAST and PSI-BLAST: a new generation of protein database search programs. Nucleic Acids Res 1997, 25(17):3389-3402.

46. Zhang Y, Skolnick J: SPICKER: A clustering approach to identify nearnative protein folds. J Comput Chem 2004, 25(6):865-871.

47. Zhang Y, Skolnick J: Scoring function for automated assessment of protein structure template quality. Proteins-Structure Function and Bioinformatics 2004, 57(4):702-710.

48. Humphrey W, Dalke A, Schulten K: VMD: visual molecular dynamics. J Mol Graph 1996, 14(1):33-38, 27-38.

doi:10.1186/1471-2105-12-208

Cite this article as: Tian et al: NCACO-score: An effective main-chain dependent scoring function for structure modeling. BMC Bioinformatics 2011 12:208 